JGG 2021;69:211-214

doi: $10.36150 / 2499-6564-N 264$

\title{
Histological confirmation of a rare case of hyperfunctioning thyroid cancer with bone metastasis in elderly patient
}

Giovanni Ruotolo ${ }^{1}$, Elvira Bonacci ${ }^{1}$, Rosa Paola Cerra ${ }^{1}$, Carmen Ruberto², Anna Maria Lavecchia ${ }^{3}$, Alberto Castagna ${ }^{2}$

${ }^{1}$ SOC Geriatrics, Azienda Ospedaliera Pugliese-Ciaccio, Catanzaro, Italy; ${ }^{2}$ Center for Cognitive Disorders and Dementia - DSS Catanzaro, ASP Catanzaro, Italy; ${ }^{3}$ SOC Pathological Anatomy, Azienda Ospedaliera Pugliese-Ciaccio, Catanzaro, Italy

Thyroid cancer is the most common malignancy of the endocrine system, with an increasing incidence in the past three decades.

The present case report describes an elderly subjects affected by follicular thyroid cancer associated with hyperthyroidism identified with a bone metastasis.

Key words: hyperthyroidism, thyroid cancer, bone metastasis, elderly patient

\section{INTRODUCTION}

Received: September 09, 2020

Accepted: April 26, 2021

\section{Correspondence}

Alberto Castagna

Center for Cognitive Disorders and Dementia DSS Catanzaro, Azienda Sanitaria Provinciale di Catanzaro, viale Crotone 88100 Catanzaro, Italy Tel./Fax +39 09617033013

E-mail: albertocastagna@tiscali.it

How to cite this article: Ruotolo G, Bonacci E, Cerra RP, et al. Histological confirmation of a rare case of hyperfunctioning thyroid cancer with bone metastasis in elderly patient. Journal of Gerontology andGeriatrics2021;69:211-214.https://doi. org/10.36150/2499-6564-N264

(C) Copyright by Società Italiana

di Gerontologia e Geriatria (SIGG)

\section{(c) (1) $(9)$}

OPEN ACCESS

This is an open access article distributed in accordance with the CC-BY-NC-ND (Creative Commons Attribution-NonCommercial-NoDerivatives 4.0 International) license. The article can be used by giving appropriate credit and mentioning the license, but only for non-commercial purposes and only in the original version. For further information: https://creativecommons.org/licenses/by-nc-nd/4.0/deed.en
Thyroid cancer is the most common malignancy of the endocrine system, with an increasing incidence in the past three decades. Clinically, affected subjects usually present euthyroidism and thyrotoxicosis is unusual and limited to case reports. In 2006 Gulcelik et al. reported 2.8\% hyperthyroidism among patients with thyroid malignancy in an iodinedeficient area ${ }^{1}$. On the other hand, except for the simultaneous onset of thyroid cancer with hyperthyroidism ${ }^{2}$ due to Graves' disease, toxic multinodular goiter or toxic adenoma, only few cases of hyperfunctioning well-differentiated thyroid cancer with metastasis have been reported.

In general, we have to consider also that bone metastasis are present in about $1-7 \%$ in papillary thyroid cancer and $7-20 \%$ in follicular thyroid cancer ${ }^{2}$ with possible lung involvement and prognosis worsening. About the bone metastases, pain, pathologic fractures, and spine compression are reported ${ }^{2}$.

Moreover, hyperfunctioning tyroid cancer may be considered as relatively rare diagnosis with delayed diagnosis timing, particularly because metastases rarely represent the initial clinical presentation.

The present case report describes an elderly subjects affected by follicular thyroid cancer associated with hyperthyroidism identified with a bone metastasis.

\section{CASE REPORT}

We observed a 79-year-old woman for about one year pain persistence in the right iliac region with subsequent appearance of inguinal swelling in the same region. The patient was unable to walk and had uncontrolled chronic 
refractory pain. She had a clinical history of high blood pressure and type 2 diabetes mellitus being treated with hydrochlorothiazide (12.5 mg/ daily), torasemide (10 mg/ daily), atenolol (25 mg/ daily), glimepiride ( $2 \mathrm{mg} /$ daily). She also reported toxic nodular goiter from the age of 58, currently being treated with metimazole (10 mg/daily). On physical examination the lower right limb was edematous, without signs of inflammation. The cardio-vascular system appears with no pathological signs differently from age.

Thyroid gland was increased in volume with large nodule in the right lobe and minor nodules in the left lobe. The abdomen was globular but treatable, painful on palpation in the groin where it was appreciated a voluminous neoformation of about $10 \mathrm{~cm}$ hard to the touch. General blood chemistry tests showed leukocytosis (Red Blood cells 12,100 mm³), hyperglycaemia (165 mg/dl), increase in alpha2 globulin (12.9\%). Oncomarkers were normal (CEA, CA15.3, CA19.9, CA125, TPA, CA50). On the chest $X$-ray a micronodular-like lung picture was shown with deviation to the left of the aerial-tracheal tape in the cervical site.

The hip, right femur and sacrococcygeal column x-ray examination highlighted the lack of appreciability of the ipsilateral ischio-pubic branch with dislocation aspect of the right femoral head. A subsequent CT of the pelvis showed right ischio-pubic osteolysis with ipsilateral hip in a site, compatible with a bone metastasis from occult tumor. To obtain a histological diagnosis of neoformation, an ultrasound-guided biopsy of the lesion was performed, the result of which showed a morphological and immunophenotypic picture of neoplastic infiltration from follicular cancer of the thyroid (Fig. 1A-C), confirmed by subsequent needle aspiration on the thyroid itself (Fig. 1D- F). The specific hormonal tests showed a thyroglobulin of $95.875 \mathrm{ng} / \mathrm{ml}$ (range for age, 0-84), $\mathrm{ft} 34.82 \mathrm{pg} / \mathrm{ml}$ (range for age 2.3-4.2), ft4 $0.84 \mathrm{ng} /$ dl (range for age 0.89-1.76), TSH $0.02 \mathrm{ulU} / \mathrm{ml}$ (range for age $0.55-4.78$ ), and calcitonin $1.7 \mathrm{ng} / \mathrm{ml}$ (range for age 0-10). Total thyroidectomy was operated and the anatomopathological examination revealed a massively invasive follicular cancer (Fig. 1F). As a result, radioactive iodine therapy was administred. During the therapeutic course, fatal cerebral ischemia arose.

\section{DISCUSSION}

Hyperthyroidism associated with a thyroid cancer is a rare condition, because low thyroid-stimulating hormone (TSH) levels can suppress the development and growth of differentiated thyroid cancer cells. The majority of nodules in patients with low TSH levels are considered as not malignancy (NCCN, British
Thyroid Association) ${ }^{3}$; however, an increasing number of thyroid cancer cases are diagnosed in patients with Graves' disease, toxic goiter and functioning thyroid adenoma. Presently, 28 cases of metastatic hyperfunctioning thyroid cancer have been described. All patients had either clinical thyrotoxicosis with biochemical data indicating hyperthyroidism, or been diagnosed as thyrotoxicosis. All patients presented with multiple or large metastases to the bone, lungs, liver or mediastinum. The mean patient age was $61.2 \pm 10.8$ years, and the female: male ratio was 1.8.

Differently from cases reported, in areas with a high incidence of Goiter and in elderly patients, as in our patient, it is necessary to consider the presence of follicular thyroid cancer in the presence of persistent skeletal pain, skeletal swelling and even occasional evidence of osteolytic lesions, as well as when pathological fractures or symptoms of bone marrow compression.

In the present case report bone metastasis have been identifying for thyrotoxicosis due to follicular thyroid cancer, clinical event to be considered truly unusual ${ }^{4-6}$. On the other hand, the tumor size may be considered as an additional relevant prognostic factor for hyperfunctioning thyroid cancer and in the present case report was $4 \mathrm{~cm}$. Again the serum thyroglobulin evaluation is important too in order to identify the residual thyroid functioning, and in the patient described the serum values were $95.875 \mathrm{ng} / \mathrm{ml}$ (reference range 0-84). Conversely, the TSH receptor gene somatic mutations may explain the hyperthyroidism caused by thyroid cancer, considering that these specific mutations tend to stimulate both the tumoral tissue growth and function for the constitutive activation of the intracellular cAMP cascade, induce hormone production and leading to hyperthyroidism. Similarly to the case reported in literature, in the case described, FT3 levels were higher than FT4 levels for unclear and unknown reasons, although the role of the iodine-deficient environment should be considered, as well as the inability of thyroid cancer cells to take up iodine and the levels of type 1 and type 2 deiodinases expression in the tumoral tissue ${ }^{7}$.

\section{CONCLUSIONS}

Rarely, neoplastic hyperthyroidism occurs and the finding of metastases is even more rare. However, patients with apparently not relevant characteristics may exhibit aggressive tumors with bone metastases at the time of diagnosis. This condition may be taken into consideration especially in areas with a high incidence of goiter and in elderly patients, suggesting the opportunity of high clinical awareness and close follow-up to decrease morbidity and mortality in these patients. 
A
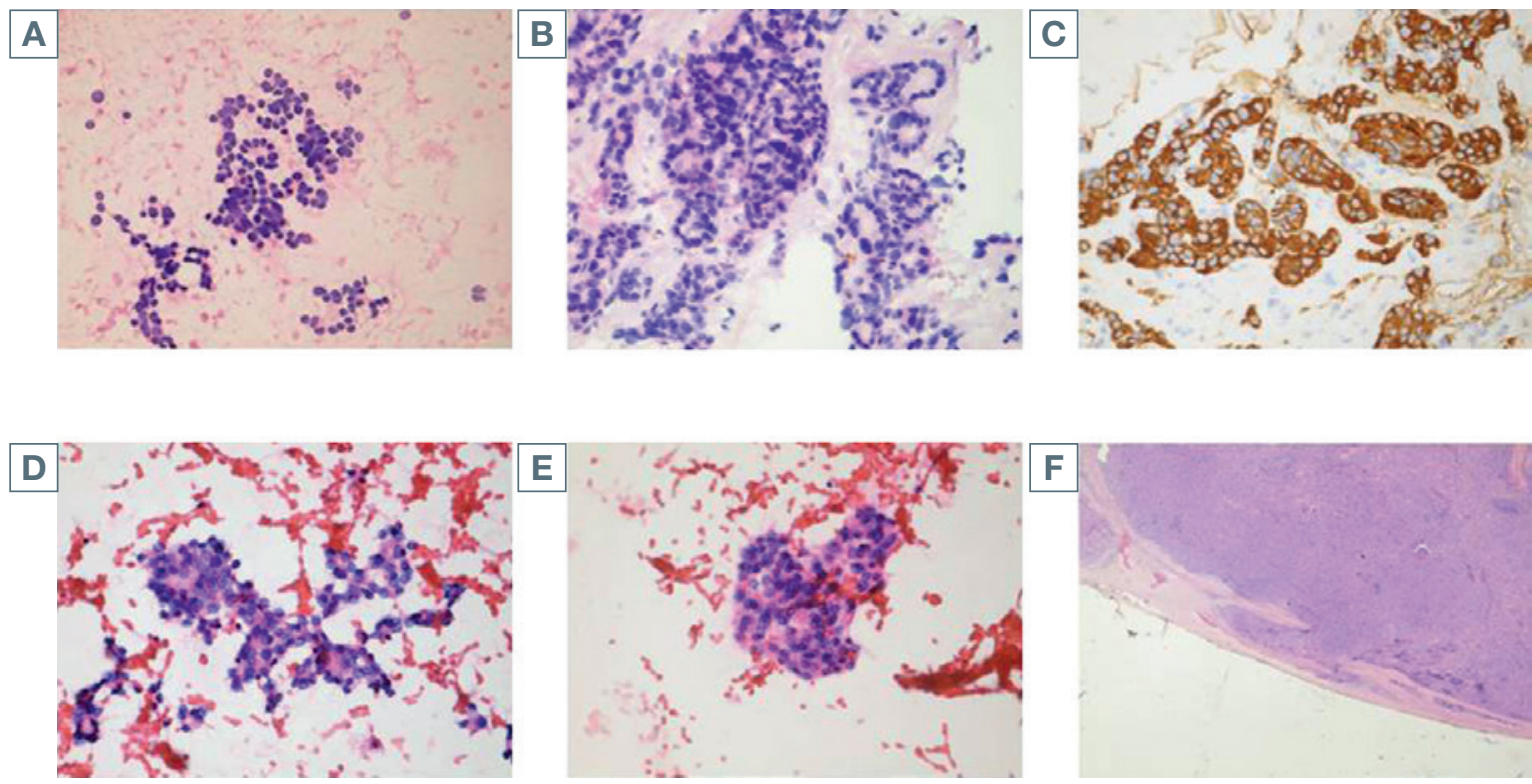

Figure 1. A) The cytologycal examination on the FNA of the tumor mass in the groin showed a cellular component consisting of small elements with hyperchromicand monomorphic nuclei in solid and acinar-like micro-aggregates; B) The histological examination on FNAB confirmed the presence of a mainly acinar epitheliomorphic growth pattern consisting of relatively monomorphic cell elements infiltrating fibrous stroma; C) The immunohistochemical investigation gave positive results for thyroglobulin, a specific marker of thyroid origin; D-E) The subsequent cytological examination on FNA of a suspected thyroid nodule confirmed the presence of a follicular neoplasm with morphological and architectural characteristics completely superimposable to those of the metastatic lesion; F) Histological examination of the nodule after total thyroidectomy allowed the diagnosis of massively invasive follicular carcinoma; the image shows an infiltration point of the tumor capsule; in the lower insert, the infiltration of a vessel is visible which, together with the capsular infiltration, represents one of the two criteria on which the diagnosis of malignancy of follicular lesions of the thyroid glandis based.

Ethical consideration

None.

\section{Acknowledgement}

None.

\section{Funding}

None.

\section{Conflict of interest}

On behalf of all authors, the corresponding author states that there is no conflict of interest.

\section{Statement of human and animal rights}

All procedures performed in studies involving human participants were in accordance with the ethical standards of the institutional and/or national research committee and with the 1964 Helsinki declaration and its later amendments or comparable ethical standards.

\section{Informed consent}

Written informed consent for publication of their clinical details and clinical images was obtained from the proxy.

\section{References}

1 Gulcelik MA, Gulcelik NE, Dinc S, et al. The incidence of hyperthyroidism in patients with thyroid cancer in an area of iodine deficiency. J Surg Oncol 2006;94:35-39. https:// doi.org/10.1002/jso.20508

2 Lin JD, Lin SF, Chen ST, et al. Long-term follow-up of papillary and follicular thyroid carcinomas with bone metastasis. PLoS One 2017;12:e0173354. https://doi.org/10.1371/ journal.pone.0173354

3 Haugen BR, Alexander EK, Bible KC, et al. 2015 American thyroid association management guidelines for adult patients with thyroid nodules and differentiated thyroid cancer: the American thyroid association guidelines task 
force on thyroid nodules and differentiated thyroid cancer. Thyroid 2016;26:1-133. https://doi.org/10.1089/ thy.2015.0020

4 Liu J, Wang YE, Da D, et al. Hyperfunctioning thyroid carcinoma: a systematic review. Mol Clin Oncol 2019;11:535550. https://doi.org/10.3892/mco.2019.1927

5 Biyi A, Zaimi S, Doudouh A. Functioning metasteses from thyroid papillary carcinoma in bone. Case report. J Nucl Med Technol 2016;44:253-254. https://doi.org/10.2967/ jnmt.116.174573
6 K Ikejiri, K, Furuyama M, Muranaka T, et al. Carcinoma of the thyroid manifested as hyperthyroidism caused by functional bone metastasis. Case report. Clin Nucl Med 1997;22:227-230. https://doi. org/10.1097/00003072-199704000-00003

7 Faivre-Defrance F, Carpentier P, Do Cao C, et al. Thyrotoxicosis revealing metastases of unrecognized thyroid cancer: a report on two cases. Case report. Ann Endocrinol (Paris) 2007;68:389-394. https://doi.org/10.1016/j. ando.2007.06.003 